\title{
Correlation between lung neoplasm and serum level of osteopontin: A meta-analysis
}

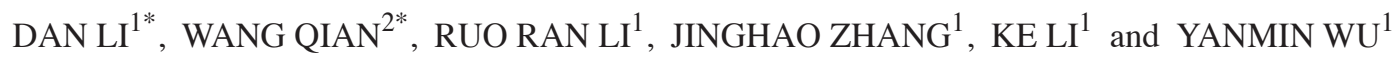 \\ Departments of ${ }^{1}$ Respiratory Medicine and ${ }^{2}$ Hematology, Xuzhou Central Hospital, The Affiliated Xuzhou \\ Hospital of Medical College of Southeast University, Xuzhou, Jiangsu 221009, P.R. China
}

Received January 5, 2016; Accepted February 29, 2016

DOI: $10.3892 /$ br.2016.619

\begin{abstract}
The aim of this meta-analysis was to evaluate the clinical significance of serum osteopontin (OPN) levels in lung neoplasm in patients to establish a novel diagnostic score model. Articles were identified by searching the PubMed, Web of Science, Google Scholar, China National Knowledge Infrastructure, and Wang Fang databases. Studies identified were pooled, and the standardized mean difference (SMD) and its corresponding 95\% confidence interval (CI) were calculated. Subgroup analyses and publication bias detection were also conducted. Version 12.0 STATA software was used for statistical analysis. A final analysis of 1,327 subjects together (740 patients with lung neoplasms and 587 controls) was performed from 10 clinical case-control studies. The meta-analysis results showed a positive association between serum OPN levels and lung neoplasm $(\mathrm{SMD}=5.59,95 \% \mathrm{CI}$ : 1.85-3.32, $\mathrm{P}<0.001)$. The subgroup analysis by ethnicity detected that high levels of serum OPN may be the main risk factor for lung neoplasms in Asians (SMD=1.76, 95\% CI: 1.24-2.29, $\mathrm{P}<0.001)$, but not in Caucasians $(\mathrm{P}=0.072)$. In conclusion, the present meta-analysis indicated that serum OPN levels were generally elevated in lung neoplasm patients, and thus, serum levels of OPN may be useful in diagnosing lung neoplasm in certain population groups.
\end{abstract}

\section{Introduction}

Lung neoplasm is the leading cause of cancer-related mortalities worldwide. Approximately $80-85 \%$ of cases are non-small-cell lung cancer. The 5-year survival rate for lung cancer is $18 \%$ (1).

Correspondence to: Dr Yanmin Wu, Department of Respiratory Medicine, Xuzhou Central Hospital, The Affiliated Xuzhou Hospital of Medical College of Southeast University, 199 South Jiefang Road, Xuzhou, Jiangsu 221009, P.R. China

E-mail: nxa980@163.com

*Contributed equally

Key words: osteopontin, lung neoplasm, meta-analysis
Even for those patients with early-stage disease who undergo surgical resection, the postoperative recurrence rate is higher than that of other types of cancer (2). Lung neoplasm has the highest mortality rate in the USA, accounting for almost 86,220 mortalities in 2010, with an estimated 116,000 new cases and 86,930 mortalities in $2014(1,3)$. It is well known that early diagnosis and treatment lead to better outcome. However, our understanding of possible factors connected with early diagnosis is limited. Thus, it is essential to identify novel and useful biological tumor markers that may more accurately establish the diagnosis of lung neoplasm. In recent years, studies have suggested that preoperative osteopontin (OPN) is positively correlated with lung neoplasm progression, which may be a clinically useful biomarker for predicting lung cancer (4-6).

OPN is a 44-kDa, secreted, highly acidic, and adhesive protein that is detected in whole body liquids (7). Accepted as the primary phosphorylated glycoprotein of the bone, OPN is simultaneously secreted in a large number of tissues and cells, including blood vessels, skin tissues, as well as immune cells (8). In clinical practice, OPN is regarded as a chemotactic factor as well as a matrix factor and a cytokine, possessing pleiotropic functions (9). OPN is involved in many physiological processes, bone remodeling, macrophage migration, angiogenesis, and neutrophil migration $(8,10)$. Nevertheless, previous findings showed that OPN is highly involved in the development of cardiovascular diseases and other types of cancer, including colorectal cancer, gastric cancer, breast cancer, and ovarian neoplasm (11-15). In addition, OPN has been found to be associated with the presence and progression of lung neoplasm, demonstrating that plasma OPN concentrations were higher in lung neoplasm patients when compared with healthy individuals (5). Previous findings have demonstrated that OPN increased the invasion of lung cancer cells by triggering ROCK signaling mediated by the FAK/PI3K/AKT pathway, which in turn induces lamellipodia formation by inactivating cofilin (16). Furthermore, increasing OPN serum levels are positively associated with advanced disease states and smoking history (6). A higher expression of OPN in NSCLC tumors was associated with poor patient outcome (17-19). Therefore, OPN may be considered a new diagnostic marker for predicting, detecting, and evaluating lung neoplasm. Additionally, OPN is a promising biomarker for lung neoplasm prediction, diagnosis, prognosis and even 
metastasis $(5,20,21)$. However, other studies have presented inconsistent results (22). Due to these conflicting results, we conducted a meta-analysis to evaluate the potential usefulness of serum OPN levels for the prediction and diagnosis of lung neoplasm.

\section{Materials and methods}

Search strategy. Related articles were identified by searching the PubMed, Web of Science, Google Scholar, China National Knowledge Infrastructure, and Wang Fang databases comprehensively for published articles that assessed the correlations between OPN serum levels and lung neoplasm for the period up to March 31, 2015, using the keywords 'Lung Neoplasms' or 'lung neoplasm' or 'lung cancers' or 'lung cancer' or 'cancer of lung' or 'lung carcinoma' or 'lung adenocarcinoma' or 'lung tumor', 'Osteopontin' or 'Sialoprotein' or 'Secreted Phosphoprotein 1' or 'Bone Sialoprotein 1' or 'Uropontin' or 'OPN'. The search was performed without language restriction. Additional potential relevant articles were retrieved through a manual search of references from original reports.

Selection criteria. Any randomized intervention case-control studies that involved the association of OPN serum levels with lung neoplasm as a primary outcome were initially taken into consideration. Aside from studies involving healthy participants as controls, studies that had patients diagnosed with lung neoplasm confirmed by histopathologic examinations and associated with OPN serum levels were also included for the initial review of the articles. Studies that did not provide the number of lung neoplasm cases or sufficient information regarding serum OPN expression levels were not included. A relatively low number of participants in a study was not considered sufficient grounds for exclusion, but all the included studies had $\geq 20$ participants. Any studies that were duplicates, lacked complete data, or had unavailable data were excluded. If the same participants were involved in more than one study, only the most recent or most complete study was included after careful re-examination.

Data extraction. To minimize the bias and improve reliability, two investigators extracted information according to the selection criteria independently and reached a consensus on all the items through discussion and re-examination. The following relevant data were extracted from eligible studies prospectively: surname of first author, year of publication, study type, study design, sample size, source of controls, age, gender, ethnicity and country of origin, and detection method of OPN serum levels. Due to subjects from different ethnicities, information was extracted separately and classified into Asians and Caucasians.

Statistical analysis. The current statistical meta-analysis was conducted utilizing a random effects model (DerSimonian and Laird method) or a fixed effects model (Mantel-Haenszel method) of individual study results when data from independent studies could be combined. The random effects model was applied when heterogeneity existed among studies, while the fixed effects model was applied when there was no statistical heterogeneity. The summary standardized mean difference
(SMD) with $95 \%$ confidence intervals (CI) was calculated for the case versus control category of OPN serum levels with the Z-test. The subgroup meta-analyses were also conducted by ethnicity and source of controls to determine the potential effect modification. Heterogeneity across the enrolled studies was evaluated using Cochran's $\mathrm{Q}$-statistic. $\mathrm{P}<0.05$ was considered to indicate statistical significance (23).

As a result of the low statistical power of Cochran's Q-statistic, the $\mathrm{I}^{2}$ test was also measured to reflect the possibility of heterogeneity between studies (24). Sensitivity analysis was performed to evaluate whether the results were potentially affected significantly by deleting single studies individually to determine the influence of individual data sets on the pooled SMD. The funnel plot was constructed to assess the effect of publication bias on the validity of the estimates. The symmetry of the funnel plot was subsequently evaluated using Egger's linear regression test (25). Tests were two-sided, and $\mathrm{P}<0.05$ was considered to indicate a statistically significant difference. STATA software, version 12.0 (Stata Corporation, College Station, TX, USA) was used for statistical analysis.

\section{Results}

Baseline characteristics of included studies. The original search yielded a total of 31 papers related to the searched keywords. The flow chart of the study selection process is provided in Fig. 1. Ten studies $(22,26-34)$ were found to meet the inclusion criteria, with the publication year ranging from 2001 to 2014. All the articles identified were case-control studies that assessed the association of OPN serum levels and lung neoplasm in Asian populations (22,26-28,31-33) ( 7 studies) and Caucasian populations $(29,30,34)$ (3 studies). The detection method applied in the present meta-analysis was enzyme-linked immunosorbent assay. Table I shows the characteristics of the enrolled studies.

OPN serum levels in lung neoplasm. Ten case-control studies referred to the OPN serum levels in lung neoplasm. The results of the correlation between the levels of OPN and lung neoplasm are shown in Fig. 2. Using the random effects model, we determined that there was heterogeneity $(\mathrm{P}<0.001)$. The meta-analysis results identified a positive association between OPN serum levels and lung neoplasm $(\mathrm{SMD}=5.59,95 \% \mathrm{CI}$ : 1.85-3.32, $\mathrm{P}<0.001)$. The subgroup analysis based on ethnicity suggested that a high level of serum OPN was the main risk factor for lung neoplasm in Asian populations $(\mathrm{SMD}=1.76$, 95\% CI: $1.24-2.29, \mathrm{P}<0.001)$, but not in Caucasian populations $(\mathrm{P}=0.072$ ) (Fig. 3). Further subgroup analyses by source of controls showed an obvious association between the levels of OPN and lung neoplasm in the population-based (PB) and hospital-based (HB) subgroups (all $\mathrm{P}<0.001)$ (Fig. 3).

Sensitivity analysis and publication bias. Sensitivity analysis showed that the statistical indications changed slightly when the study of Zhou et al (22) or Yin and Zhang (31) was omitted. Therefore, the current meta-analysis data were inconsistent. The graphical funnel plots of the 10 studies are a little asymmetrical, and Egger's test shows a publication bias in the current meta-analysis $(\mathrm{t}=6.38, \mathrm{P}<0.001)$ (data not shown). 
Table I. Characteristics of included studies focused on protein expression of OPN.

\begin{tabular}{|c|c|c|c|c|c|}
\hline Author (year) & Ethnicity & Source of controls & Sample size (case/control) & Method & Refs. \\
\hline Song (2014) & Asians & $\mathrm{PB}$ & $32 / 35$ & ELISA & (26) \\
\hline Qiu and Fu (2012) & Asians & $\mathrm{PB}$ & $56 / 40$ & ELISA & $(27)$ \\
\hline Xu et al (2012) & Asians & $\begin{array}{l}\text { PB } \\
\mathrm{HB}\end{array}$ & $\begin{array}{l}102 / 30 \\
102 / 42\end{array}$ & $\begin{array}{l}\text { ELISA } \\
\text { ELISA }\end{array}$ & $(28)$ \\
\hline Karadag et al (2011) & Caucasians & PB & $63 / 25$ & ELISA & (29) \\
\hline Zhou et al (2011) & Asians & $\mathrm{HB}$ & $\begin{array}{r}56 / 45 \\
103 / 45\end{array}$ & $\begin{array}{l}\text { ELISA } \\
\text { ELISA }\end{array}$ & (22) \\
\hline Blasberg et al (2010) & Caucasians & $\mathrm{HB}$ & $60 / 56$ & ELISA & $(30)$ \\
\hline Yin and Zhang (2009) & Asians & $\begin{array}{l}\mathrm{PB} \\
\mathrm{HB}\end{array}$ & $\begin{array}{l}80 / 40 \\
80 / 40\end{array}$ & $\begin{array}{l}\text { ELISA } \\
\text { ELISA }\end{array}$ & $(31)$ \\
\hline Dong et al (2008) & Asians & $\begin{array}{l}\mathrm{PB} \\
\mathrm{HB}\end{array}$ & $\begin{array}{l}120 / 49 \\
120 / 23\end{array}$ & $\begin{array}{l}\text { ELISA } \\
\text { ELISA }\end{array}$ & $(32)$ \\
\hline Weng and Fu (2007) & Asians & $\begin{array}{l}\text { PB } \\
\mathrm{HB}\end{array}$ & $\begin{array}{l}48 / 40 \\
48 / 45\end{array}$ & $\begin{array}{l}\text { ELISA } \\
\text { ELISA }\end{array}$ & (33) \\
\hline Fedarko et al (2001) & Caucasians & PB & $20 / 77$ & ELISA & (34) \\
\hline
\end{tabular}

PB, population-based; HB, hospital-based; ELISA, enzyme-linked immunosorbent assay.

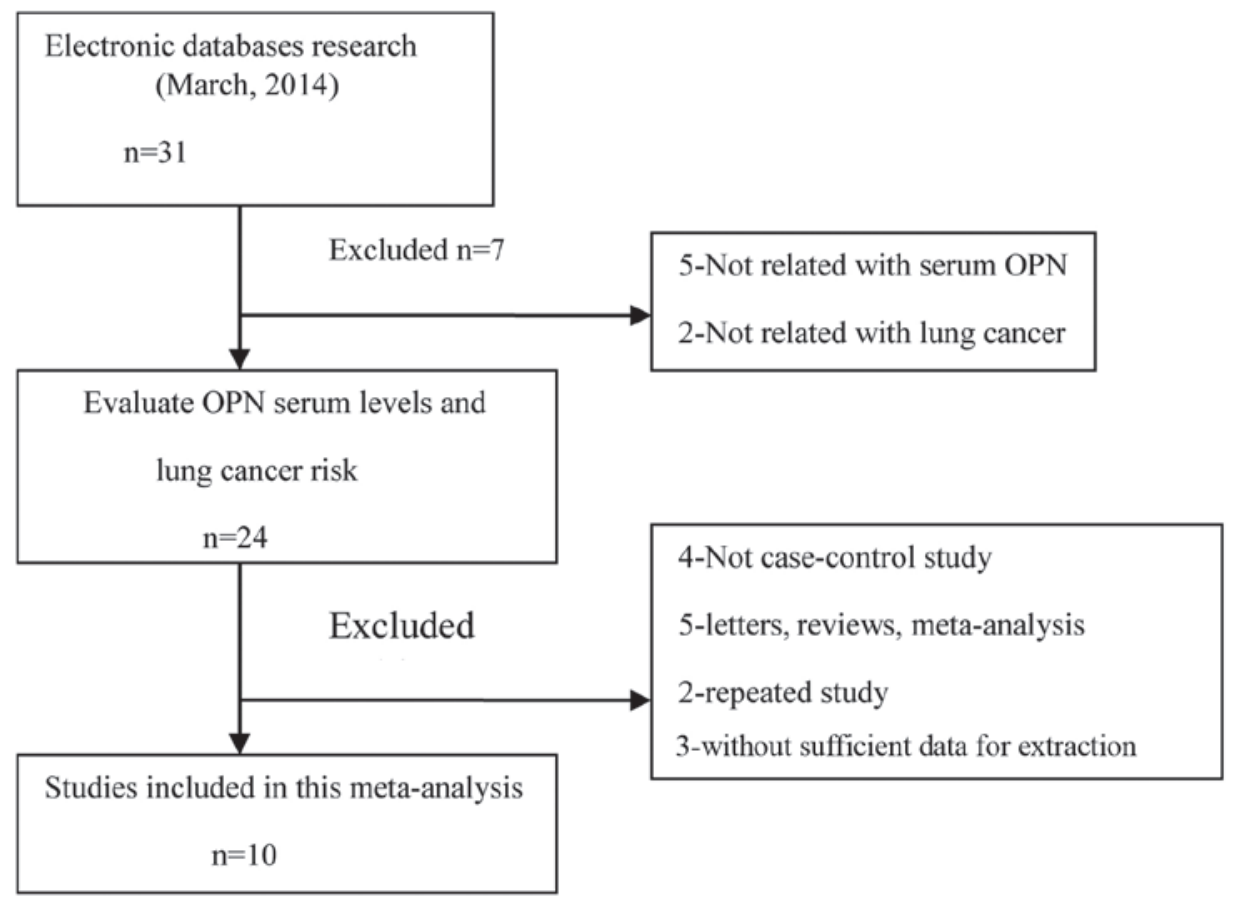

Figure 1. Study plan. OPN, osteopontin.

\section{Discussion}

In this meta-analysis, we investigated the possible relationship between the serum levels of OPN and the development of lung neoplasm. Our results suggest that lung neoplasm patients have higher OPN serum levels, demonstrating that OPN serum levels may be important in the development of lung neoplasm. Previous findings have shown that there was a significant reverse correlation between OPN and overall and disease-free survival (20). Generally, OPN, an integrin-binding protein, is involved in numerous signaling pathways, such as cancer cell growth, adhesion, apoptosis, metastasis, proliferation, migration, invasion, and angiogenesis $(7,35,36)$. OPN is capable of inducing cell invasion by markedly increasing the expression of Rho-associated kinase 1 (ROCK1), an upstream effector of LIMK/cofilin mediated by the FAK/PI3K/AKT pathway. ROCK1 inactivated cofilin by phosphorylating LIMK and cofilin which block lamellipodia formation. Thus, the invasion of lung neoplasm cells can be increased by OPN under stress condi- 


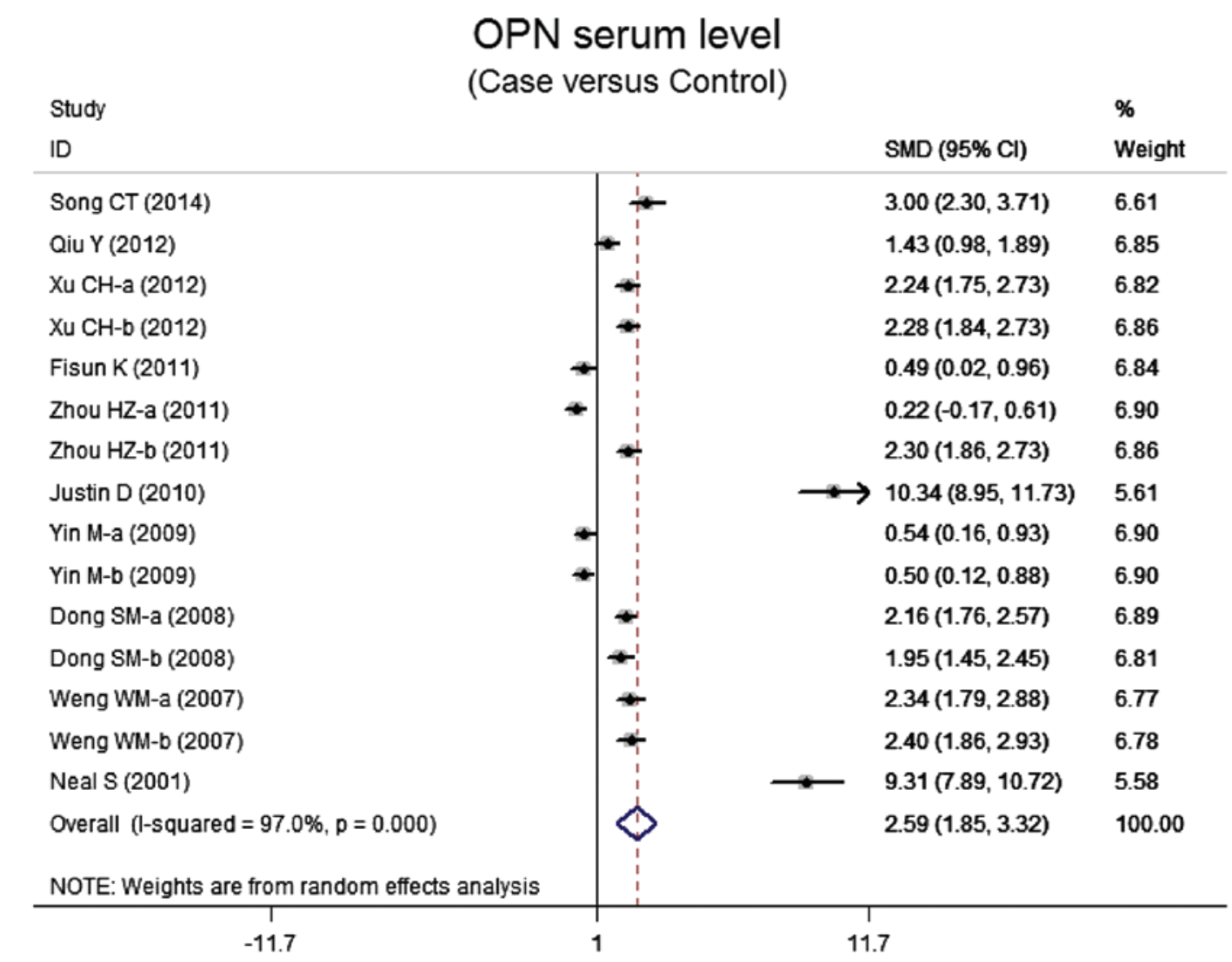

Figure 2. Forest plot for the relationship between serum levels of osteopontin and lung neoplasm patients. OPN, osteopontin; SMD, standardized mean difference; CI, confidence interval; ID, identity.

tions via the FAK/PI3K/AKT pathway and ROCK1 expression induction (16). Furthermore, OPN is involved in lung neoplasm angiogenesis because OPN expression in cancer tissue was positively correlated with microvascular density (37). In addition, the OPN level was correlated with $\alpha \mathrm{v}$ integrin expression and the decreased apoptotic activity of lung adenocarcinomas cells (38). OPN also led to an obvious promotion of in vitro invasion and in vivo lung metastasis by increasing the levels of matrix metalloproteinase- 2 and urokinase plasminogen activator (39). Thus, higher levels of OPN promotes tumor progression through invasion, angiogenesis, metastasis and inhibition of apoptosis. In concordance with our results, Zhang et al (40) identified that higher levels of OPN were positively associated with stage, lymph node metastasis, tumor size and pathology of lung neoplasms because of their anti-apoptotic or proliferative effects on lung neoplasm. Thus, OPN is a possible biomarker for lung neoplasm detection, prognosis, and even their apeutic intervention.

Given the fact that other factors may influence the relationship between serum OPN level and lung neoplasm, we conducted a stratified analysis based on ethnicity and source of controls. The subgroup analysis by ethnicity showed that higher serum levels of OPN were evident in Asians, while no similar result was observed among Caucasian populations. One possible explanation may be the different genetics and environments of the two ethnicities. Additionally, there was no obvious effect of source of controls on the relationship between OPN and ovarian neoplasm. Thus, higher serum levels of OPN remain strongly connected with the progression of ovarian neoplasm. This lends further credence to the creditability of the analysis as it indicates that the samples are random and well selected. Therefore, our results were partially consistent with the hypothesis that higher serum levels of OPN may have a strong connection with lung neoplasm, suggesting that the serum OPN level is an optimal marker for lung neoplasm identification and prognosis.

Limitations of this meta-analysis should be considered. First, possible selection biases were identified, there was a highly significant heterogeneity among the 10 evaluable articles. Additionally, the Egger's test showed a publication bias in our meta-analysis. Second, is that certain unpublished articles and abstracts were not taken into account as their data were not available, leading to potential publication bias in the present study. Language may also introduce a bias. Specifically, English or Chinese studies were selected while excluding qualified studies in other languages. A third potential limitation is that our meta-analysis was weakened by an inability to extract the original data from the included studies. Despite the above limitations, this is the first systematic review on the association of serum OPN levels with the risk of lung neoplasm. Application of a statistical approach to combine the results from multiple studies in this meta-analysis and to achieve strong results led to the research methods being carried out using strict inclusion and exclusion criteria, indicating the validity and significance of our conclusion.

In summary, the present meta-analysis indicates that increased serum levels of OPN may contribute to an 
A

OPN serum level

(Ethnicity:Case versus Control)

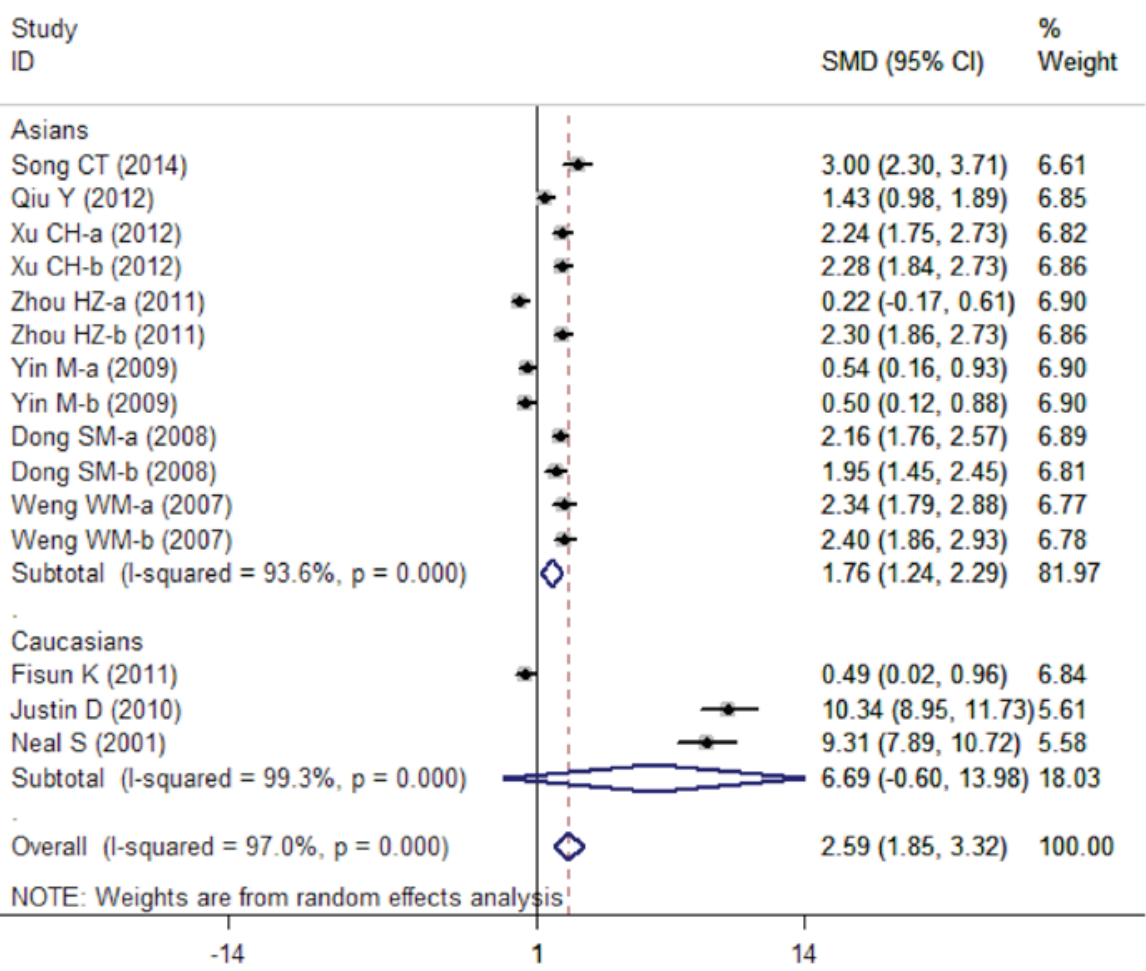

B

OPN serum level

(Source of controls:Case versus Control)

\begin{tabular}{|c|c|c|c|}
\hline \multicolumn{2}{|l|}{$\begin{array}{l}\text { Study } \\
\text { ID }\end{array}$} & \multirow[t]{2}{*}{$\operatorname{SMD}(95 \% \mathrm{Cl})$} & \multirow[t]{2}{*}{$\begin{array}{l}\% \\
\text { Weight }\end{array}$} \\
\hline Population-based & & & \\
\hline Song CT (2014) & & $3.00(2.30,3.71)$ & 6.61 \\
\hline Qiu Y (2012) & +1 & $1.43(0.98,1.89)$ & 6.85 \\
\hline $\mathrm{Xu} \mathrm{CH}-\mathrm{a}(2012)$ & + & $2.24(1.75,2.73)$ & 6.82 \\
\hline Fisun K (2011) & & $0.49(0.02,0.96)$ & 6.84 \\
\hline Yin M-a (2009) & & $0.54(0.16,0.93)$ & 6.90 \\
\hline Dong SM-a (2008) & -1 & $2.16(1.76,2.57)$ & 6.89 \\
\hline Weng WM-a (2007) & + & $2.34(1.79,2.88)$ & 6.77 \\
\hline Neal S (2001) & $\longrightarrow$ & $9.31(7.89,10.72)$ & 5.58 \\
\hline Subtotal $(1-$ squared $=96.5 \%, p=0.000)$ & & $2.52(1.55,3.49)$ & 53.27 \\
\hline \multicolumn{4}{|l|}{ Hospital-based } \\
\hline Xu CH-b (2012) & + & $2.28(1.84,2.73)$ & 6.86 \\
\hline Zhou HZ-a (2011) & & $0.22(-0.17,0.61)$ & 6.90 \\
\hline Zhou HZ-b (2011) & + & $2.30(1.86,2.73)$ & 6.86 \\
\hline Justin D (2010) & $\longrightarrow$ & $10.34(8.95,11.73)$ & 5.61 \\
\hline Yin M-b (2009) & & $0.50(0.12,0.88)$ & 6.90 \\
\hline Dong SM-b (2008) & +1 & $1.95(1.45,2.45)$ & 6.81 \\
\hline Weng WM-b (2007) & + & $2.40(1.86,2.93)$ & 6.78 \\
\hline Subtotal $(1-$ squared $=97.7 \%, p=0.000)$ & & $2.69(1.49,3.89)$ & 46.73 \\
\hline Overall $(1-$ squared $=97.0 \%, p=0.000)$ & $\infty$ & $2.59(1.85,3.32)$ & 100.00 \\
\hline \multicolumn{4}{|l|}{ NOTE: Weights are from random effects analysis? } \\
\hline \begin{tabular}{r|r|} 
& 11.7 \\
-11.7
\end{tabular} & 11. & & \\
\hline
\end{tabular}

Figure 3. (A and B) Subgroup analyses for the relationship between the serum levels of osteopontin and lung neoplasm patients. OPN, osteopontin; SMD, standardized mean difference; CI, confidence interval; ID, identity.

aggressive progression of lung neoplasm. Based on the data obtained, OPN is a potentially useful marker for diagnosing lung neoplasms. Hoverer, in light of the limited sample size included and the potential for bias in the current study, additional investigations on serum OPN levels and lung neoplasms are needed. 


\section{References}

1. Siegel R, Ma J, Zou Z and Jemal A: Cancer statistics, 2014. CA Cancer J Clin 64: 9-29, 2014

2. Chansky K, Sculier JP, Crowley JJ, Giroux D, Van Meerbeeck J and Goldstraw P; International Staging Committee and Participating Institutions: The International Association for the Study of Lung Cancer Staging Project: Prognostic factors and pathologic TNM stage in surgically managed non-small cell lung cancer. J Thorac Oncol 4: 792-801, 2009.

3. Jemal A, Siegel R, Xu J and Ward E: Cancer statistics, 2010. CA Cancer J Clin 60: 277-300, 2010.

4. Hu Z, Lin D, Yuan J, Xiao T, Zhang H, Sun W, Han N, Ma Y, Di X, Gao M, et al: Overexpression of osteopontin is associated with more aggressive phenotypes in human non-small cell lung cancer. Clin Cancer Res 11: 4646-4652, 2005.

5. Han SS, Lee SJ, Kim WJ, Ryu DR, Won JY, Park S and Cheon MJ: Plasma osteopontin is a useful diagnostic biomarker for advanced non-small cell lung cancer. Tuberc Respir Dis (Seoul) 75: 104-110, 2013.

6. Chang YS, Kim HJ, Chang J, Ahn CM, Kim SK and Kim SK: Elevated circulating level of osteopontin is associated with advanced disease state of non-small cell lung cancer. Lung Cancer 57: 373-380, 2007.

7. Moszynski R, Szubert S, Szpurek D, Michalak S and Sajdak S: Role of osteopontin in differential diagnosis of ovarian tumors. J Obstet Gynaecol Res 39: 1518-1525, 2013.

8. Vetrone SA, Montecino-Rodriguez E, Kudryashova E, Kramerova I, Hoffman EP, Liu SD, Miceli MC and Spencer MJ: Osteopontin promotes fibrosis in dystrophic mouse muscle by modulating immune cell subsets and intramuscular TGF-beta. J Clin Invest 119: 1583-1594, 2009.

9. Cho HJ, Cho HJ and Kim HS: Osteopontin: A multifunctional protein at the crossroads of inflammation, atherosclerosis, and vascular calcification. Curr Atheroscler Rep 11: 206-213, 2009.

10. Lund SA, Giachelli CM and Scatena M: The role of osteopontin in inflammatory processes. J Cell Commun Signal 3: 311-322, 2009.

11. Okyay K, Tavil Y, Sahinarslan A, Tacoy G, Turfan M, Sen N, Gurbahar O, Boyaci B, Yalcin R, Demirkan D, et al: Plasma osteopontin levels in prediction of prognosis in acute myocardial infarction. Acta Cardiol 66: 197-202, 2011.

12. Song G, Ouyang G, Mao Y, Ming Y, Bao S and Hu T: Osteopontin promotes gastric cancer metastasis by augmenting cell survival and invasion through Akt-mediated HIF-1alpha up-regulation and MMP9 activation. J Cell Mol Med 13 (8B): 1706-1718, 2009.

13. Xu YY,Zhang YY, Lu WF, Mi YJ and Chen YQ: Prognostic value of osteopontin expression in breast cancer: A meta-analysis. Mol Clin Oncol 3: 357-362, 2015.

14. Yuan SM, Wang J, Huang HR and Jing H: Osteopontin expression and its possible functions in the aortic disorders and coronary artery disease. Rev Bras Cir Cardiovasc 26: 173-182, 2011.

15. Wang YD, Chen H, Liu HQ and Hao M: Correlation between ovarian neoplasm and serum levels of osteopontin: A meta-analysis. Tumour Biol 35: 11799-11808, 2014.

16. Kang CG, Han HJ, Lee HJ, Kim SH and Lee EO: Rho-associated kinase signaling is required for osteopontin-induced cell invasion through inactivating cofilin in human non-small cell lung cancer cell lines. Bioorg Med Chem Lett 25: 1956-1960, 2015.

17. Rud AK, Boye K, Oijordsbakken M, Lund-Iversen M, Halvorsen AR, Solberg SK, Berge G, Helland A, Brustugun OT and Mælandsmo GM: Osteopontin is a prognostic biomarker in non-small cell lung cancer. BMC Cancer 13: 540, 2013.

18. Sun BS, Li Y, Zhang ZF, You J and Wang CL: Osteopontin combined with CD44v6, a novel prognostic biomarker in non-small cell lung cancer undergoing curative resection. Ann Thorac Surg 96: 1943-1951, 2013.

19. Peng B, Wang YH, Huang Z, Feng SJ and Wang YS: Prognostic significance of osteopontin in patients with lung cancer: A meta-analysis. Int J Clin Exp Med 7: 4616-4626, 2014.
20. Yan $\mathrm{CH}$, Lv M, Li H, Song $\mathrm{X}$, Yan F, Cao S and Ren $\mathrm{X}$ : Osteopontin is a novel prognostic biomarker in early-stage non-small cell lung cancer after surgical resection. J Cancer Res Clin Oncol 141: 1371-1378, 2015.

21. Karapanagiotou EM, Terpos E, Dilana KD, Alamara C, Gkiozos I, Polyzos A and Syrigos KN: Serum bone turnover markers may be involved in the metastatic potential of lung cancer patients. Med Oncol 27: 332-338, 2010.

22. Zhou $\mathrm{H}$, Wu T and Zhu Y: The clinical significance of serum osteopontin in diagnosis of lung cancer. J Radioimmunol 24: 458-459, 2011 (In Chinese).

23. Jackson D, White IR and Riley RD: Quantifying the impact of between-study heterogeneity in multivariate meta-analyses. Stat Med 31: 3805-3820, 2012.

24. Peters JL, Sutton AJ, Jones DR, Abrams KR and Rushton L: Comparison of two methods to detect publication bias in meta-analysis. JAMA 295: 676-680, 2006.

25. Zintzaras E and Ioannidis JP: HEGESMA: Genome search meta-analysis and heterogeneity testing. Bioinformatics 21: 3672-3673, 2005.

26. Song C: Clinical significance of determination of changes CEA, SA, OPN, and VEGF levels after operative in patients with lung cancer. Frontier Med 15: 75-76, 2014.

27. Qiu Y and Fu Z: Clinical significance of determination on serum levels of vascular endothelial growth factor per platelet count and osteopontin patients with locally advanced non-small cell lung cancer (unpublished $\mathrm{PhD}$ thesis). Hebei Medical University, Shijiazhuang, R734.2, 2012.

28. Xu C, Yu K and Wang Q: The levels and clinical significance of osteopontin in serum of patients with lung cancer. J Chin Physician 14: 179-181, 2012.

29. Karadag F, Gulen ST, Karul AB, Kilicarslan N, Ceylan E, Kuman NK and Cildag O: Osteopontin as a marker of weight loss in lung cancer. Scand J Clin Lab Invest 71: 690-694, 2011.

30. Blasberg JD, Pass HI, Goparaju CM, Flores RM, Lee S and Donington JS: Reduction of elevated plasma osteopontin levels with resection of non-small-cell lung cancer. J Clin Oncol 28: 936-941, 2010.

31. Yin M and Zhang J: Study on OPN levels of serum in non-small cell lung cancer. Ningxia Med J 31: 679-680, 2009.

32. Dong S, Shao Z, Pan Y, Dai J and Chen G: The clinical significance of detection of serum osteopontin in patient with lung cancer. Mod J Integr Tradit Chin West Med 17: 3740-3741, 2008.

33. Weng $\mathrm{W}$ and Fu C: Value of serum osteopontin determination in patient with lung carcinoma. Lab Med Clin 4: 1025-1026, 2007.

34. Fedarko NS, Jain A, Karadag A, Van Eman MR and Fisher LW: Elevated serum bone sialoprotein and osteopontin in colon, breast, prostate, and lung cancer. Clin Cancer Res 7: 4060-4066, 2001.

35. Chambers AF and Vanderhyden BC: Ovarian cancer biomarkers in urine. Clin Cancer Res 12: 323-327, 2006.

36. Lin CK, Chao TK, Lai HC and Lee HS: LMX1A as a prognostic marker in ovarian mucinous cystadenocarcinoma. Am J Clin Pathol 137: 971-977, 2012

37. Yu TT, Han ZG, Shan L, Tao J, Zhang T, Yuan SF and Shen HL: Expression of osteopontin in non-small cell lung cancer and correlative relation with microvascular density. Asian Pac J Cancer Prev 15: 29-32, 2014.

38. Štemberger C, Matušan-Ilijaš K, Avirović M, Bulat-Kardum L, Ivančić A, Jonjić $\mathrm{N}$ and Lučin K: Osteopontin is associated with decreased apoptosis and $\alpha \mathrm{v}$ integrin expression in lung adenocarcinoma. Acta Histochem 116: 222-229, 2014.

39. Sun BS, You J, Li Y, Zhang ZF and Wang CL: Osteopontin knockdown suppresses non-small cell lung cancer cell invasion and metastasis. Chin Med J (Engl) 126: 1683-1688, 2013.

40. Zhang T, Zhang DM, Zhao D, Hou XM, Liu XJ, Ling XL and Ma SC: The prognostic value of osteopontin expression in non-small cell lung cancer: A meta-analysis. J Mol Histol 45: 533-540, 2014. 